\title{
Ultra-Wideband Source Localization Using a Particle-Swarm-Optimized Capon Estimator from a Frequency-Dependent Channel Modeling Viewpoint
}

\author{
Yifan Chen \\ Positioning and Wireless Technology Centre, Nanyang Technological University, 50 Nanyang Drive, Research TechnoPlaza, \\ BoderX Block, Singapore 637553 \\ Email: eychen@pmail.ntu.edu.sg \\ Vimal K. Dubey \\ Positioning and Wireless Technology Centre, Nanyang Technological University, 50 Nanyang Drive, Research TechnoPlaza, \\ BoderX Block, Singapore 637553 \\ Email:evkdubey@ntu.edu.sg \\ Received 22 June 2004; Revised 21 September 2004; Recommended for Publication by Petar Djuric

\begin{abstract}
We introduce a realistic frequency-dependent channel model for ultra-wideband (UWB) communication systems and develop a generalized broadband Capon spatial spectrum estimator for localization of multiple incoherently distributed scattering clusters. The proposed estimator is able to address the three crucial features of practical UWB impulse propagation: presence of local scattering for multiple incoherently distributed clusters, wideband array signals, and frequency-dependent dispersive effects. The particle-swarm optimization, which is a recently invented high-performance optimizer based on the movement and intelligence of swarms, is then implemented to perform a multidimensional parameter search to jointly estimate the source central angles, the polynomial regression coefficients for angle spreads, and the frequency-dependence of various clusters. Numerical experiments are also carried out to examine the performance of the algorithm under various environments and model mismatches.
\end{abstract}

Keywords and phrases: array processing, UWB channel modeling, broadband Capon estimator, particle-swarm optimization, multidimensional search.

\section{INTRODUCTION}

The emerging ultra-wideband (UWB) technology demands much research effort to achieve improved operational capabilities and cost-effective system solutions for broad commercial and military applications [1]. Theoretical study in the area of signal modeling and signal processing is thus essential for the development and performance analysis of practical UWB systems. Despite that a large volume of literature has been devoted to handle problems like source localization and sidelobe reduction for the narrowband case, fundamental differences exist between the classical array signal processing techniques, pertaining to the incidence of narrowband signals, and the problem of interest here, where the signals have large fractional bandwidth $[2,3,4,5]$.

UWB system is at present defined by the Federal Communications Commission (FCC) as any wireless transmission scheme that occupies a large fractional bandwidth over $20 \%$, or more than $500 \mathrm{MHz}$ of absolute bandwidth. Recent
UWB indoor channel measurements showed that physical paths were clustered around transmit/receive directions and each cluster corresponded to a major path to the receiver $[5,6,7,8]$. Arrivals within clusters are likely the result of closely associated objects that are part of a very similar group of paths to the receiver (e.g., reflection from walls, floors and ceilings, scattering from furniture and displays, diffraction from doorway openings, transmission through soft partitions $[8,9])$. Furthermore, each cluster, corresponding to an individual scattering center, will have its own impulse response or frequency transfer characteristics depending on the scattering mechanism $[10,11,12,13,14,15]$. For UWB impulses, various diffractions and reflections pertain to different frequency-dependent behaviors. For example, consider a UWB system operating at a frequency of $5 \mathrm{GHz}$ with a bandwidth of $2.5 \mathrm{GHz}$. In this frequency band, the difference in scattering properties is significant for a sphere/cylinder of radius $0.1 \mathrm{~m}$ [16], which thus verifies that signal components with different operating frequencies will likely be processed 
with different fidelity, dispersion, and loss. ${ }^{1}$ On the other hand, study of propagation through different materials also capitalizes the statement of spectrum dependency. For example, cloth partition shows higher loss caused by support elements inside the partitions as reported in [9]. Attenuation constants for the door, wood, and structure wood sample are also presented in [9], which sheds more light on the effects of various materials on the filtering of UWB pulses. To sum up, the UWB system structure features three distinct characteristics: presence of large angle spreads for multiple incoherently distributed scattering clusters, wideband array signals, and frequency-dependent dispersive effects.

Motivated by these facts, in this paper, we extend the simple yet accurate generalized Capon estimator for narrowband stationary signals [17] to UWB scenarios based on a realistic signal/channel model in the angular-frequency domain. The particle-swarm optimization (PSO) $[18,19]$, which is a robust stochastic evolutionary computation technique and is new to the signal processing community, is then implemented to perform the multidimensional search for the parametric setting of the underlying channel model. Some preliminary results have been presented in [20] while a more comprehensive discussion will be provided in the current paper. The paper is organized as follows. In Section 2, we present the analytical model in angular frequency domain of UWB impulse waveforms, and provide the formulation of the generalized broadband Capon estimator. Based on this estimation method, a detailed explanation of the PSO algorithm for our problem of interest is presented in Section 3. Computer simulation results are presented in Section 4, and conclusions are drawn in Section 5. The following notations will be used in this paper. I is the identity matrix. $[\cdot]^{*},[\cdot]^{H}$, and $[\cdot]^{T}$ denote complex conjugate, Hermitian, and transpose operations, respectively. $E[\cdot]$ is the expectation operator.

\section{SIGNAL AND CHANNEL MODELS FOR UWB TRANSMISSION SCHEMES}

For simplicity, we assume that the distributed signals are on the same plane as the array (i.e., a two-dimensional propagation) and the total fields are dominated by the scattered fields. By zooming out the granularity of the channel description and characterizing the channel by a set of arrayindependent parameters, the angular-frequency domain expression of the channel response can be formulated as

$$
h(f, \theta)=\sum_{k=1}^{K} \sum_{l \in \delta_{k}} \beta_{k l} e^{j \phi_{k l}} e^{-j 2 \pi f \tau_{k l}} A_{k l}\left(f, \theta_{k l}\right) \delta\left(\theta-\theta_{k l}\right),
$$

where $K$ is the total number of the scattering centers (clusters) and $s_{k}$ denotes the set of propagation paths (point

\footnotetext{
${ }^{1}$ The heightened sensitivity of UWB signals to different scatterers leads to signal-to-noise ratio (SNR) degradation at a RAKE or autocorrelation receiver output as shown in $[13,14]$.
}

source components) in the $k$ th cluster. $\delta(\cdot)$ is the delta function. For each point source, we model it using four parameters $\left\{\beta_{k l} e^{j \phi_{k l}} ; A_{k l} ; \theta_{k l} ; \tau_{k l}\right\}$ corresponding to complex amplitude; frequency-dependence; direction of arrival; path delay time.

To make the procedure discussed above feasible, we further assume that the scattering centers must have welldefined frequency-dependencies as given by their leadingorder geometry theory of diffraction (GTD) scattering coefficients. We will consider a special form of $A_{k l}\left(f, \theta_{k l}\right) \propto f^{\alpha_{k l}}$ for common structures $[10,11,12,13,14]$. For example, a ray coming from a line-of-sight (LOS) path has $\alpha_{k l}=0$ and a ray coming from a wall edge has $\alpha_{k l}=-0.5$. If the plane wave is diffracted at an endpoint or tip such as the desk corner, $\alpha_{k l}=-1$. It is further assumed that the $K$ diffuse sources and the point scattering components within each source are independent of each other. This assumption is often used to simplify calculations in many applications [21] and has been experimentally shown to be relevant to the indoor channel [5].

If these point source components are densely clustered together, we replace each of the point sources by a density function spreading over a small angular volume $\Delta \theta$ for any frequency component $f$. Equation (1) can thus be reformulated $\mathrm{as}^{2}$

$$
\begin{aligned}
& h(f, \theta)= \\
& \lim _{\Delta \theta \rightarrow 0} \sum_{k=1}^{K} \sum_{l \in S_{k}} \beta_{k} e^{j \phi_{k}} e^{-j 2 \pi f \tau_{k}} f^{\alpha_{k}} \sqrt{\rho_{k}\left(f, \theta, \gamma_{k}\right) \Delta \theta} \cdot \delta\left(\theta-\theta_{k l}\right),
\end{aligned}
$$

where $\rho_{k}\left(f, \theta, \gamma_{k}\right)$ is the power azimuthal spectrum (PAS) characterizing the distribution of the $k$ th cluster at each $f$. $\theta \in \Theta_{k}(f)$ where $\Theta_{k}$ is the region for the $k$ th scattering center within which the diffuse signal spreads and is frequencydependent [6]. $y_{k}$ is a parameter vector used to define the location of the $k$ th signal. Recently, Intel Corporation has performed several UWB wireless channel measurements in both non-line-of-sight (NLOS) and LOS scenarios, at both office and residential environments. The frequency was swept from 2 to $8 \mathrm{GHz}$ with resolution of $3.75 \mathrm{MHz}$. It has been shown that the average cluster angle spread (AS) decreased with increasing frequency as depicted in Figure 1, which was due to the fact that an electromagnetic wave of higher frequency attenuated more severely after passing through or bouncing off objects in the environment and channel became less diffuse at higher frequency. In this paper, we attempt to describe the AS variation by polynomial regression models, that is,

$$
\sigma_{\theta, k}(f)=\sum_{m=0}^{\mathcal{O}_{k}} c_{m, k} f^{m} \quad(\mathrm{GHz})
$$

\footnotetext{
${ }^{2}$ The subscript $l$ has been dropped in (2) to indicate that $\beta_{k}, \phi_{k}, A_{k}$, and $\tau_{k}$ correspond to the strongest path in the $k$ th cluster assuming a symmetric density function $\rho_{k}\left(f, \theta, \gamma_{k}\right)$.
} 


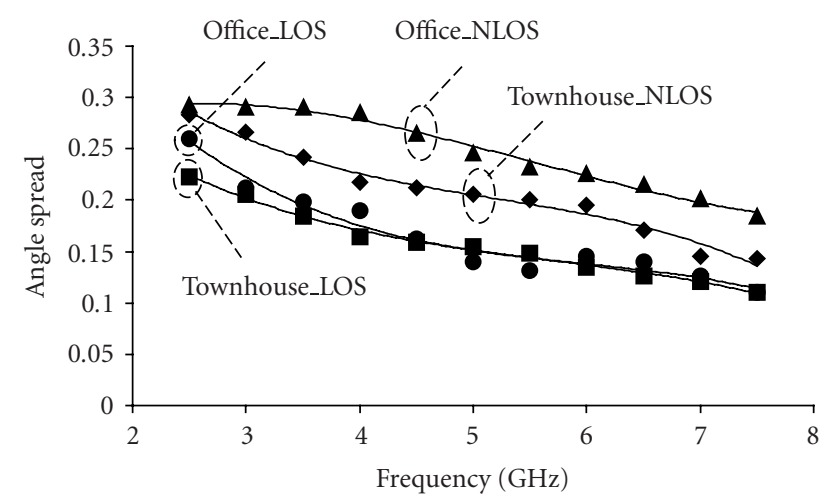

FIGURE 1: Average cluster angle spreads in the townhouse and office environments [6] and their polynomial regression curves.

where $\mathcal{O}_{k}$ is the order of the polynomial equation for the $k$ th cluster and $c_{m, k}$ 's are the corresponding coefficients. Figure 1 also depicts fitting of the empirical data to four third-order polynomial equations, for which reasonable agreement is justified for all the four groups of data. As we further increase the polynomial order, finer curve fitting can be obtained. Nevertheless, increasing model order will significantly raise the computational load without much improvement in the underlying model structure. Following from the above arguments, the parameter vector $\boldsymbol{y}_{k}$ is described as $\left[\underline{\theta}_{k}, c_{0, k}, \ldots, c_{\mathcal{O}_{k}, k}\right]$ with $\underline{\theta}_{k}$ being the mean direction of arrival (DOA) of the $k$ th cluster. The most common shapes suggested for the PAS function $\rho_{k}\left(f, \theta, \gamma_{k}\right)$ include uniform [22], Gaussian [22], and Laplacian [5, 6, 7], depending on the propagation environments.

Consider that the signals of $K$ scattering centers impinge on an array of $q$ sensors. The complex envelope of the array output can be expressed as

$$
\begin{aligned}
& \mathbf{y}(t)=\sum_{k=1}^{K} \mathbf{s}_{k}(t)+\mathbf{n}(t) \\
&=\sum_{k=1}^{K} \int_{f_{\min }}^{f_{\max }} \int_{\theta \in \Theta_{k}(f)} \beta_{k} e^{j \phi_{k}} e^{-j 2 \pi f \tau_{k}} f^{\alpha_{k}} \\
& \quad \times \sqrt{\rho_{k}\left(f, \theta, \boldsymbol{\gamma}_{k}\right) d \theta} e^{j 2 \pi f(t+\boldsymbol{\tau}(\theta))} d \nu(f)+\mathbf{n}(t),
\end{aligned}
$$

where $\mathbf{y}(t)$ is the $q \times 1$ array snapshot vector, $\mathbf{s}_{k}(t)$ is the $q \times 1$ vector that describes the contribution of the $k$ th scattering center to the array output, and $\mathbf{n}(t)$ is the vector of sensor noise. $\left[f_{\min }, f_{\max }\right]$ is the spectral support ( $3 \mathrm{~dB}$ cutoff points) of the signal, and $d \nu(f)$ denotes a measure of the signal spectrum at $f . \boldsymbol{\tau}(\theta)$ is a $q \times 1$ vector representing the propagation delay corresponding to a DOA $\theta$ with respect to a given reference sensor. For the case of a uniform linear array, $\boldsymbol{\tau}(\theta)=$

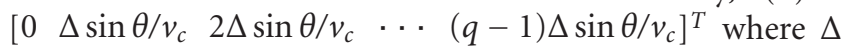
is the interelement spacing and $v_{c}$ is the speed of electromagnetic waves. Assuming uncorrelated DOAs, the $\left(q_{1}, q_{2}\right)$ th element of the spatial covariance matrix of the received signal from the $k$ th cluster can be expressed as

$$
\begin{gathered}
{\left[\mathbf{R}_{k}\left(\alpha_{k}, \gamma_{k}\right)\right]_{q_{1}, q_{2}}} \\
=\int_{f_{\min }}^{f_{\max }} \int_{f_{\min }}^{f_{\max }} \int_{\theta \in \Theta_{k}(f)} \beta_{k}^{2} e^{-j 2 \pi\left(f-f^{\prime}\right) \tau_{k}}\left(f \cdot f^{\prime}\right)^{\alpha_{k}} \rho_{k}\left(f, \theta, \gamma_{k}\right) \\
\quad \times e^{j 2 \pi f \tau_{q_{1}}(\theta)-j 2 \pi f^{\prime} \tau_{q_{2}}(\theta)} d \theta E\left[d \nu(f) d \nu^{*}\left(f^{\prime}\right)\right] .
\end{gathered}
$$

We further make the common assumption that the spectral components at different frequencies are mutually orthogonal and the typical UWB pulse is the Gaussian monocycle $[1,5]$, which has been widely used for analytical evaluation of UWB systems. Hence,

$$
E\left[d \nu(f) d v^{*}\left(f^{\prime}\right)\right] \triangleq S(f)=A f^{4} T^{6} \exp \left(-\pi f^{2} T^{2}\right) \delta\left(f-f^{\prime}\right),
$$

where $S(f)$ denotes the power spectral density of the source [5], $A$ is a normalization constant, and $T$ is a parameter determining the temporal width of the pulse. Casting (6) into (5) yields

$$
\begin{aligned}
& {\left[\mathbf{R}_{k}\left(\alpha_{k}, \boldsymbol{\gamma}_{k}\right)\right]_{q_{1}, q_{2}}} \\
& =\int_{f_{\min }}^{f_{\max }} \int_{\theta \in \Theta_{k}(f)} A \beta_{k}^{2} T^{6} f^{2 \alpha_{k}+4} \\
& \quad \times \exp \left\{j 2 \pi f \frac{\left(q_{1}-q_{2}\right) \Delta}{v_{c}} \sin \theta-\pi f^{2} T^{2}\right\} \\
& \quad \times \rho_{k}\left(f, \theta, \boldsymbol{\gamma}_{k}\right) d \theta d f .
\end{aligned}
$$

In the cases of uniform, Gaussian, and Laplacian distributions, $\rho_{k}\left(f, \theta, \gamma_{k}\right)$ takes the forms of

$$
\begin{aligned}
\rho_{k}\left(f, \theta, \boldsymbol{\gamma}_{k}\right)=Q_{\mathrm{U}, k} \cdot\left\{\varepsilon\left[\theta-\left(\underline{\theta}_{k}-\frac{\sigma_{\theta, k}(f)}{2}\right)\right]\right. \\
\left.-\varepsilon\left[\theta-\left(\underline{\theta}_{k}+\frac{\sigma_{\theta, k}(f)}{2}\right)\right]\right\}, \\
\rho_{k}\left(f, \theta, \boldsymbol{\gamma}_{k}\right)=Q_{\mathrm{G}, k} \cdot \frac{2}{\sigma_{\theta, k}(f) \sqrt{2 \pi}} \exp \left[-\frac{2\left(\theta-\underline{\theta}_{k}\right)^{2}}{\sigma_{\theta, k}^{2}(f)}\right], \\
\rho_{k}\left(f, \theta, \boldsymbol{\gamma}_{k}\right)=Q_{\mathrm{L}, k} \cdot \frac{\sqrt{2}}{\sigma_{\theta, k}(f)} \exp \left[-\frac{2 \sqrt{2}\left|\theta-\underline{\theta}_{k}\right|}{\sigma_{\theta, k}(f)}\right],
\end{aligned}
$$

respectively, where $\varepsilon[\cdot]$ is the step function and $Q_{\mathrm{U}, k}, Q_{\mathrm{G}, k}$, and $Q_{\mathrm{L}, k}$ are normalization constants. Assuming the additive noise at each sensor to be white Gaussian, the overall covariance matrix is given by

$$
\mathbf{R}_{y}=\mathbf{R}_{s}(\boldsymbol{\alpha}, \boldsymbol{\gamma})+\sigma^{2} \mathbf{I}=\sum_{k=1}^{K} \mathbf{R}_{k}\left(\alpha_{k}, \boldsymbol{\gamma}_{k}\right)+\sigma^{2} \mathbf{I}
$$

where $\mathbf{R}_{y}=E\left\{\mathbf{y}(t) \mathbf{y}^{H}(t)\right\}$ is the array covariance matrix and $\mathbf{R}_{s}(\boldsymbol{\alpha}, \boldsymbol{\gamma})=E\left\{\mathbf{s}(t) \mathbf{s}^{H}(t)\right\}$ is the overall covariance matrix of the signals from the multiple incoherently distributed scattering centers with $\boldsymbol{\alpha}=\left[\alpha_{1}, \alpha_{2}, \ldots, \alpha_{K}\right]$ and $\boldsymbol{\gamma}=\left[\boldsymbol{\gamma}_{1}\right.$, $\left.\gamma_{2}, \ldots, \gamma_{k}\right]$. 
A generalized Capon DOA estimator for narrowband sources has been proposed in [17] and shows a substantially improved performance relative to several popular spread source localization techniques such as the DISPARE algorithm [23] and the root-MUSIC-based estimator [24]. We will further extend this method to UWB scenarios as presented below. The minimum variance distortionless response problem is formulated as

$$
\min _{\mathbf{w}} \mathbf{w}^{H} \mathbf{R}_{y} \mathbf{w} \quad \text { subject to } \mathbf{w}^{H} \mathbf{R}_{s}(\boldsymbol{\Lambda}) \mathbf{w}=1 .
$$

Following from (12), the proposed spatial filter maintains distortionless spatial response to multiple hypothetical sources with the parameter vector $\Lambda=[\boldsymbol{\alpha}, \boldsymbol{\gamma}]$ while maximally rejecting the contribution of any other sources. Such a response is now represented by means of the covariance matrix $\mathbf{R}_{s}(\boldsymbol{\Lambda})$ and thus the distortionless response is maintained in (12) in the mean power sense rather than in the deterministic sense. According to the derivations in [17], the minimal value of the objective function $\mathbf{w}^{H} \mathbf{R}_{y} \mathbf{w}$ is equal to the smallest generalized eigenvalue of the matrix pencil $\left\{\mathbf{R}_{y}, \mathbf{R}_{s}(\boldsymbol{\Lambda})\right\}$, and the generalized Capon pseudospectrum is defined as the beamformer output power when the beamformer is steered to sources with the parameter $\boldsymbol{\Lambda}$. The generalized Capon estimator is thus given by

$$
P(\boldsymbol{\Lambda})=\lambda_{\min }\left\{\mathbf{R}_{y}, \mathbf{R}_{s}(\boldsymbol{\Lambda})\right\}=\frac{1}{\sigma_{\max }\left\{\mathbf{R}_{y}^{-1} \mathbf{R}_{s}(\boldsymbol{\Lambda})\right\}},
$$

where $\lambda_{\min }\{\cdot\}$ stands for the minimum generalized eigenvalue of a matrix pencil and $\sigma_{\max }\{\cdot\}$ is the maximum eigenvalue of a matrix. The parametric vector $\boldsymbol{\Lambda}$ can then be estimated from the global maximum of (13). Apparently, it involves a multidimensional search, which can hardly be solved by traditional nonlinear programming techniques. This motivates us to resort to more powerful evolutionary algorithms. In the discussions that follow, instead of (13), we will reformulate the objective function in a different manner and thus change it into a constrained minimization problem

$$
\begin{aligned}
& \text { minimize } o(\boldsymbol{\Lambda})=\frac{1}{P(\boldsymbol{\Lambda})}=\sigma_{\max }\left\{\mathbf{R}_{y}^{-1} \mathbf{R}_{s}(\boldsymbol{\Lambda})\right\}, \quad \boldsymbol{\Lambda} \in \boldsymbol{\Omega}, \\
& \text { subject to } \sum_{m=0}^{\mathcal{O}_{k}} c_{m, k} f_{\min }^{m} \leq \sigma_{\theta \max }, \quad \sum_{m=0}^{\mathcal{O}_{k}} c_{m, k} f_{\max }^{m} \geq \sigma_{\theta \min }, \\
& \qquad \sum_{m=1}^{\mathcal{O}_{k}} m c_{m, k} f^{m-1} \leq 0,
\end{aligned}
$$

where $\boldsymbol{\Omega}$ is usually defined by upper and lower bounds of the decision variable $\Lambda$. The constraints are imposed to ensure that the angle spread is limited within the range $\left[\sigma_{\theta \min }, \sigma_{\theta \max }\right]$ over a predefined frequency range, $\zeta f \triangleq\left[f_{\min }, f_{\max }\right]$, and it decreases monotonically as the frequency increases.

\section{PARTICLE-SWARM OPTIMIZATION FOR LOCATING THE GLOBAL MINIMUM}

In 1995, Kennedy and Eberhart proposed the PSO algorithm inspired from the simulation of social behavior of flocking organisms, such as swarms of birds and fish schools $[18,19]$. Since its invention, PSO has gained increasing popularity because it is simple in concept and easy to implement. Previous study has shown the PSO to be an effective alternative to more established evolutionary algorithms for certain kinds of problems [25, 26, 27]. Furthermore, the particle-swarm paradigm retains the conceptual simplicity of genetic algorithm (GA) while being subject to only one operator greatly reduces the complexity of applying a generic optimization routine to an arbitrary problem. This technique has been successfully applied to many optimization applications such as tackling multiobjective problems [28], minimax problems [29], integer programming problems [29], and many engineering applications $[25,26]$.

PSO can be understood by imaging a swarm of birds that search for food in an open field. Without any a priori knowledge of the field, the individual birds spread out and begin their search in random locations. Each bird can remember the location where it found the most of the food, and share this information with its neighbors. As time goes on, the birds profit from the discoveries and previous experience of all other companions during the search for food by exploring the field in this manner: continuously updating their speed and direction of travel depending on how successful at finding food they have been in comparison to the rest of the swarm. Constantly, they are checking the field they fly over against previously encountered locations of highest food concentration. Eventually, the whole field is probed and the birds end up swarming around the location with the globally highest concentration of food.

In a mathematical formulation, each companion, called particle or agent, is treated as a point in a $D$-dimensional solution space which adjusts its own flying according to its flying experience as well as the experience of other particles. We represent the $i$ th particle by the $D$-dimensional vector $\mathbf{x}_{i}=$ $\left[x_{i 1}, x_{i 2}, \ldots, x_{i D}\right]$ and the globally best particle in the swarm is denoted by the position vector gbest $=\left[g_{1}, g_{2}, \ldots, g_{D}\right]$. The best previous position of the $i$ th particle is recorded and represented as pbest $_{i}=\left[p_{i 1}, p_{i 2}, \ldots, p_{i D}\right]$, and the velocity of the $i$ th particle is $\mathbf{v}_{i}=\left[v_{i 1}, v_{i 2}, \ldots, v_{i D}\right]$. The particles evolve according to the equations

$$
\begin{gathered}
v_{i d}=w v_{i d}+c_{1} r_{1}\left(p_{i d}-x_{i d}\right)+c_{2} r_{2}\left(g_{d}-x_{i d}\right), \\
x_{i d}=x_{i d}+v_{i d},
\end{gathered}
$$

where $d=1,2, \ldots, D ; i=1,2, \ldots, I$, and $I$ is the population size. $w$ is the inertial weight. $c_{1}$ and $c_{2}$ are two positive constants. $r_{1}$ and $r_{2}$ are two random values in the range $[0,1]$. The first equation is used to calculate the $i$ th particle's new velocity by taking into account three terms: the particle's previous velocity, the distance between its best previous and current positions, and the distance between the position 
of the best particle in the swarm and the ith particle's current position. In general, the performance of each particle is measured according to a problem-dependent fitness function. The inertia weight $w$ determines to what extent the particle remains along its original course unaffected by the pull of pbest or gbest. It regulates the tradeoff between the exploration and exploitation abilities of the swarm. A large $w$ tends to encourage global exploration as a result of the particle being less moved by the pull of pbest and gbest, whereas a small one tends to facilitate local exploitation in that particles being rapidly pulled toward pbest and gbest. A general rule of thumb suggests that it is better to initially set $w$ to a large value, in order to make a better global exploration of the search space, and gradually decrease it to get finer solutions. $c_{1}$ and $c_{2}$ are scaling factors that determine the relative pull of pbest and gbest. These are also referred to as the cognitive and social rates, respectively. $c_{1}$ determines how much the particle is influenced by the memory of its best location, and $c_{2}$ is a factor determining how much the particle is influenced by the rest of the swarm. As default values, $c_{1}=c_{2}=2$ were proposed in [19], although experimental results indicated that alternative combinations sometimes led to superior performance [30].

This algorithm limits the resulting velocity to a maximum allowed velocity $v_{\max }$. Eberhart et al. noted that the PSO performed better if $v_{\max }$ in each dimension was set equal to the dynamic range of that dimension. To further ensure that the particles are always confined within the solution space, the rejecting strategy is implemented in this paper: the particles are allowed to fly without any physical constriction. However, particles that travel outside the allowed solution space are not evaluated for fitness (i.e., assigned with a sufficiently large fitness value for our minimization problem) so that the swarm is repelled away from those infeasible regions. This technique helps to save computation time by evaluating what is in the feasible region, while not interfering with the natural motion of the swarm.

To define a reasonable solution space (i.e., specification of maximum and minimum values for each dimension) for the DOA estimation problem, we propose the following algorithm to determine the range of polynomial regression coefficients.

Step 0. Define a reasonable range for angle spread, $\sigma_{\theta 1}$, such that $\sigma_{\theta 1} \in \Omega_{\theta} \triangleq\left[\sigma_{\theta \min }, \sigma_{\theta \max }\right]$ over the spectral support, $\zeta f$, for the concerned environment type.

Step 1. Discretize the spectral support $\zeta f$ into a set of grid points with equal separation, denoted as the vector $\boldsymbol{\zeta} \mathbf{f}=$ $\left[f_{1}, f_{2}, \ldots, f_{N}\right]$.

Step 2. Randomly generate the first angle spread $\sigma_{\theta 1}$ corresponding to $f_{1}$. Note that $\sigma_{\theta 1}$ is uniformly distributed in $\Omega_{\theta}$.

Step 3. Randomly generate the second angle spread $\sigma_{\theta 2}$ corresponding to $f_{2}$. Note that $\sigma_{\theta 2}$ is uniformly distributed in $\left[\sigma_{\theta \min }, \sigma_{\theta 1}\right]$, which is to ensure that the angle spread is always non-increasing as the frequency increases.

Step 4. Repeat Step 3 for $\sigma_{\theta 3}, \sigma_{\theta 4}, \ldots, \sigma_{\theta N}$ and denote the vector $\boldsymbol{\sigma}_{\boldsymbol{\theta}}=\left[\sigma_{\theta 1}, \sigma_{\theta 2}, \ldots, \sigma_{\theta N}\right]$.
Step 5. Perform the $\mathcal{O}$ th-order polynomial regression for the two sets of data, $\zeta$ f and $\sigma_{\theta}$.

Step 6. Repeat Steps 2 to 5 for several runs.

Step 7. Based on the simulation results, choose the upper and lower bounds of the search space for each dimension. Sometimes the obtained range may be further divided into several subdivisions to assist the searching process.

In addition, the range for the central angle $\underline{\theta}$ and frequency-dependence $\alpha$ can be easily determined.

To alleviate the problem that PSO may end up finding local minima and fail to locate the global minimum in the landscape, we can apply the "deflection," "stretching," and "repulsion" techniques [31] to transform the original objective function $o(\boldsymbol{\Lambda})$ immediately after a local minimum $\boldsymbol{\Lambda}_{i}$ has been detected. For this approach, the most fundamental question is how to identify a local minimum. Parsopoulos and Vrahatis [29] used a threshold value $\kappa$ such that when $o\left(\boldsymbol{\Lambda}_{i}\right) \leq \kappa$ for the $i$ th particle, the particle is isolated from the swarm and marked as a potential global solution. However, $\kappa$ depends strongly on the a priori knowledge of the global minimum as well as the objective function's landscape and dimensionality, which cannot be availed for our DOA estimation problem. Therefore, we could apply another approach by monitoring changes in the fitness of a particle. We track the standard deviation in particle $i$ 's fitness, $\varsigma_{i}$, over several iterations. When $\varsigma_{i}$ is smaller than a threshold value $\delta$, a minimum is identified. $\delta$ is a more intuitive and tunable parameter than $\kappa$ and is less problem-dependent. Another difficulty is that most stochastic optimization algorithms including PSO and GA suffer from the curse of dimensionality, which simply implies that the performance deteriorates as the dimensionality of the search space increases. This phenomenon may arise in UWB source localization problems when there is a large number of scattering centers, each of them exhibiting a high-order AS variation pattern. A possible solution to overcome this problem is to employ the cooperative PSO (CPSO) through using multiple swarms to optimize different components of the solution vector cooperatively [32]. And each component corresponds to a single scattering center. The entire algorithm described in this section is shown pictorially in Figure 2.

\section{EXPERIMENTAL RESULTS}

In the following examples, we consider a uniform linear array of 10 sensors with a sensor spacing of half-wavelength corresponding to the center frequency of the band. The spectral support of the UWB source is taken to be the interval [ $3 \mathrm{GHz}, 7 \mathrm{GHz}$ ], representing a fractional bandwidth of $80 \%$, and the pulse duration $T=0.25$ nanosecond. In all the experiments, the SNR is set to $30 \mathrm{~dB}$. Also note that the sufficiently large fitness value applied in the rejecting strategy is $10^{5}$.

Experiment 1 (uniform PAS). In this experiment, we only consider a single-cluster scenario for the indoor UWB channel and assume that the DOAs from point source components are uniformly distributed over the angular range with 


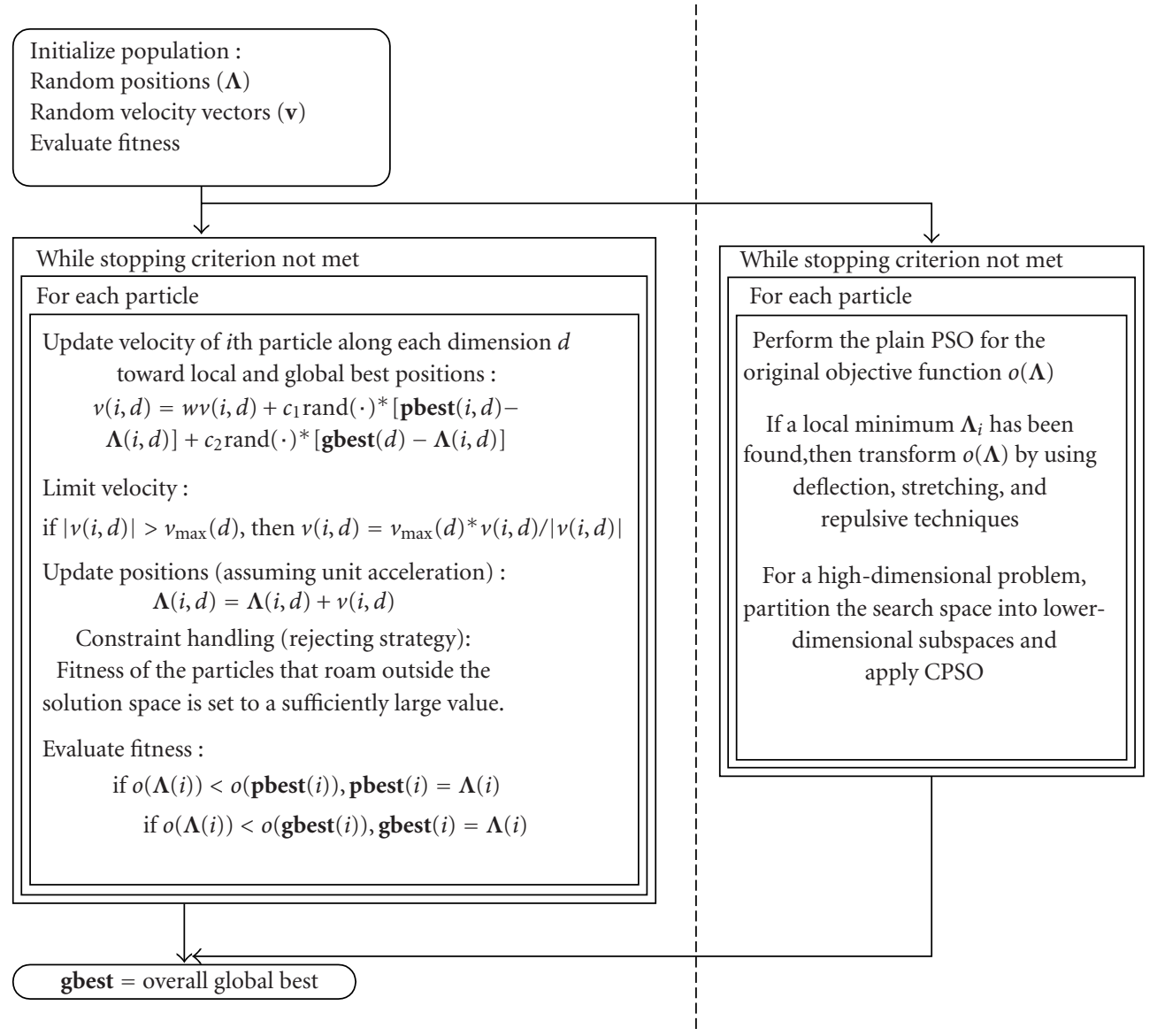

FIGURE 2: Flowchart illustrating a plain PSO (left-hand figure) and a modified PSO (right-hand figure).
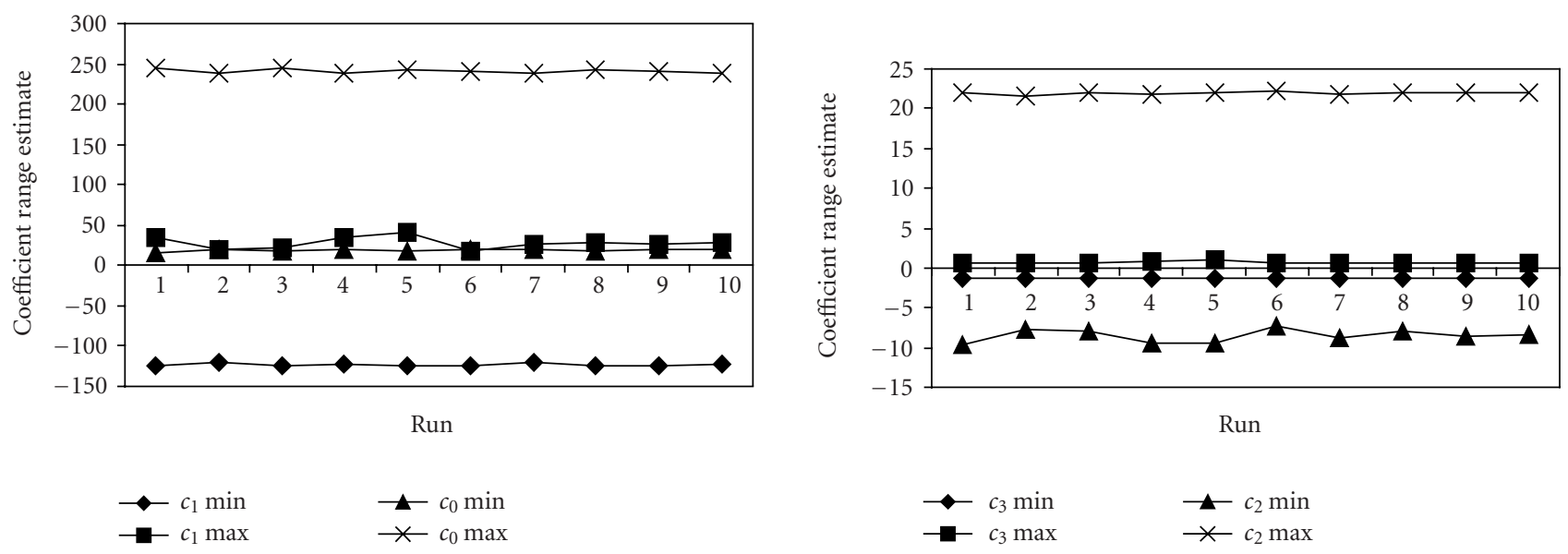

Figure 3: Coefficient range estimate based on the algorithm presented in Section $3\left(\sigma_{\theta \min }=20^{\circ}\right.$ and $\sigma_{\theta \max }=70^{\circ}$ over an extended spectral support).

a central angle $\underline{\theta}=\pi / 6$. We further assume that the underlying model structure of the concerned environment type takes the form of a third-order polynomial, and the frequencydependence of the propagation mode can be described by $\alpha=-0.5$. Subsequently, the parameter vector to be estimated is assumed to be $[\pi / 6,0.06,-1.2,2.5,50,-0.5]$ in a 6 - dimensional hypercube and the fitness function is defined by (14). A population size of 500 is selected, $c_{1}$ and $c_{2}$ are both set to 2.0 , and the inertial weight $w$ is varied linearly from 0.9 to 0.4 over 500 iterations. The range in which the optimal solution is searched for is obtained by applying the algorithm presented in Section 3 and depicted in Figure 3. 


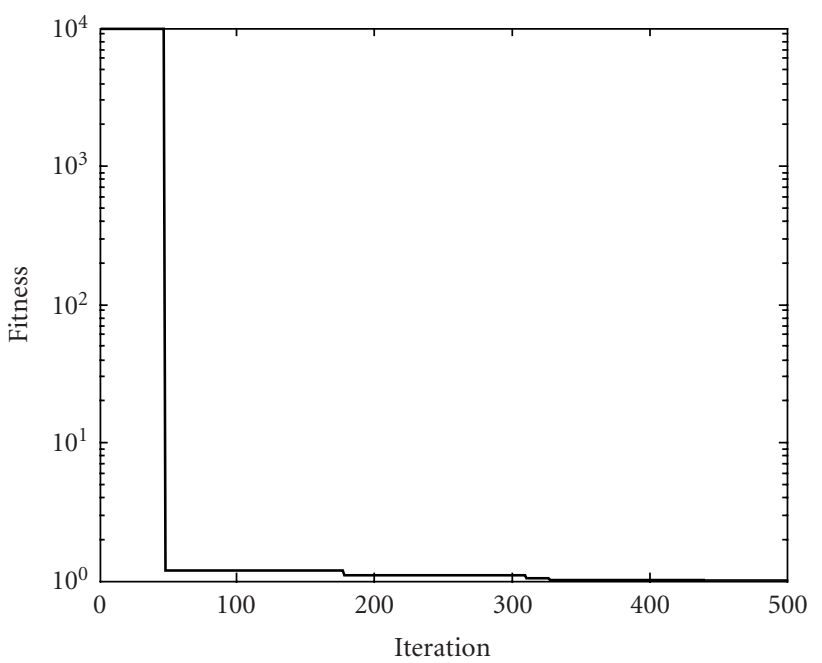

(a)

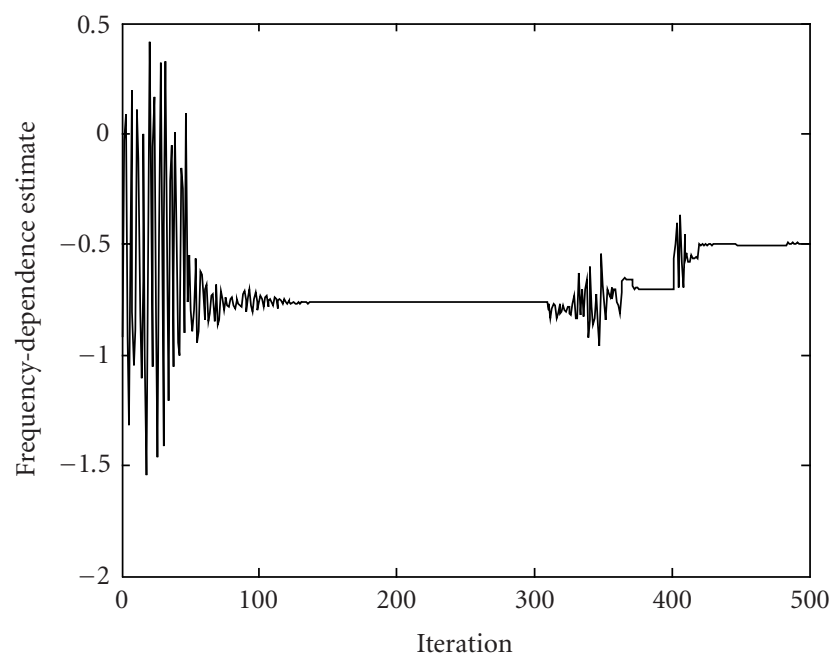

(c)

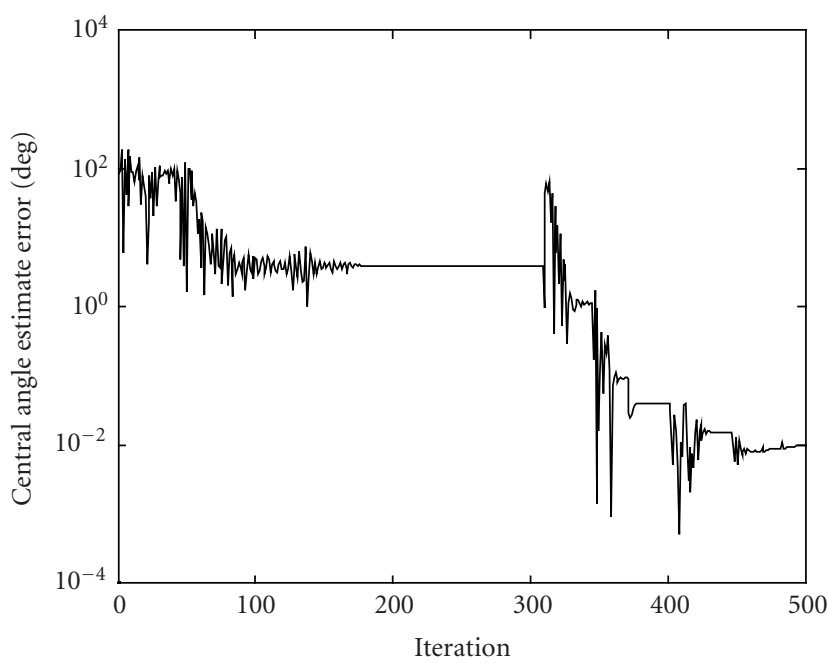

(b)

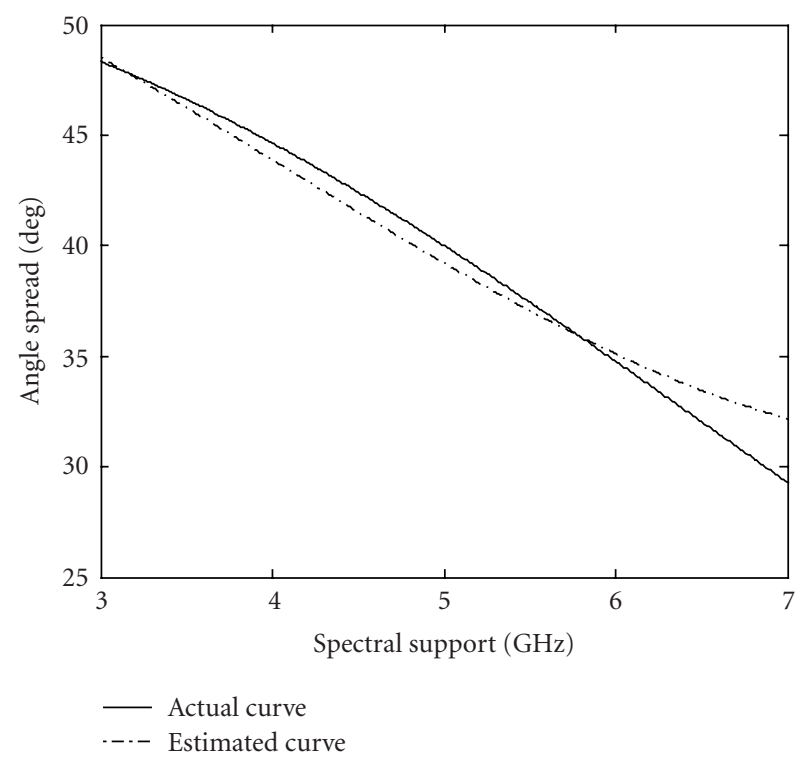

(d)

FIGURE 4: Convergence curves of (a) calculated fitness of the PSO algorithm, (b) central angle estimate error, (c) frequency-dependence estimate, and (d) the actual AS variation curve over the entire spectral support versus the estimated curve at the 500th iteration for Experiment 1 (uniform PAS).

It is instructive to examine the calculated fitness value over 500 iterations to graphically show how quickly the PSO algorithm can converge to a solution of the problem. From Figure $4 \mathrm{a}$, it is clear that the particles start to swarm around the feasible locations at around the 50th iteration. Figure $4 \mathrm{~b}$ shows the central angle estimate error versus the iteration number. As it can be seen from the figure, the estimation performance is satisfactory (about $0.01^{\circ}$ ) and indeed the result is even better than the original generalized Capon estimator for narrowband array signals with much narrower angle spreads $\left(4^{\circ}\right.$ and $\left.5^{\circ}\right)$ presented in [17]. Figure 4c shows that the frequency-dependence converges to the actual value $(\alpha=-0.5)$ at around the 420th iteration and the final result is -0.4953 . Finally, the two curves (the estimated AS variation curve and the actual one) are shown in Figure 4d where the estimated curve is obtained at the 500th iteration, demonstrating that PSO can successfully find the polynomial regression coefficients. Nevertheless, several other runs show that the plain PSO sometimes can detect suboptimal solutions. These results signify a need to implement the modify PSO to provide a way of escape from the local minima when the convergence stalls.

Experiment 2 (Gaussian PAS). In this experiment the channel model structure and the PSO parameter setting are identical to the previous example except that the signal is now 


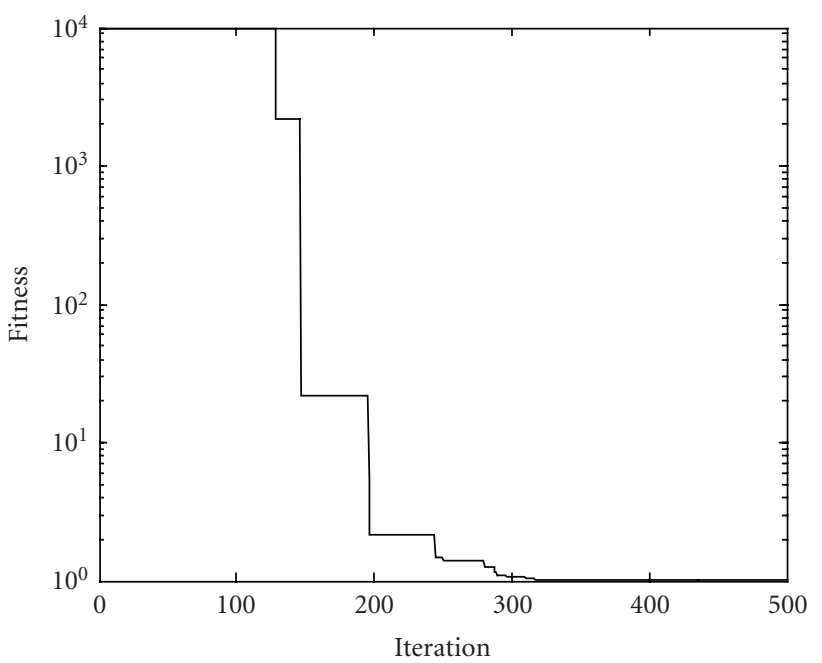

(a)

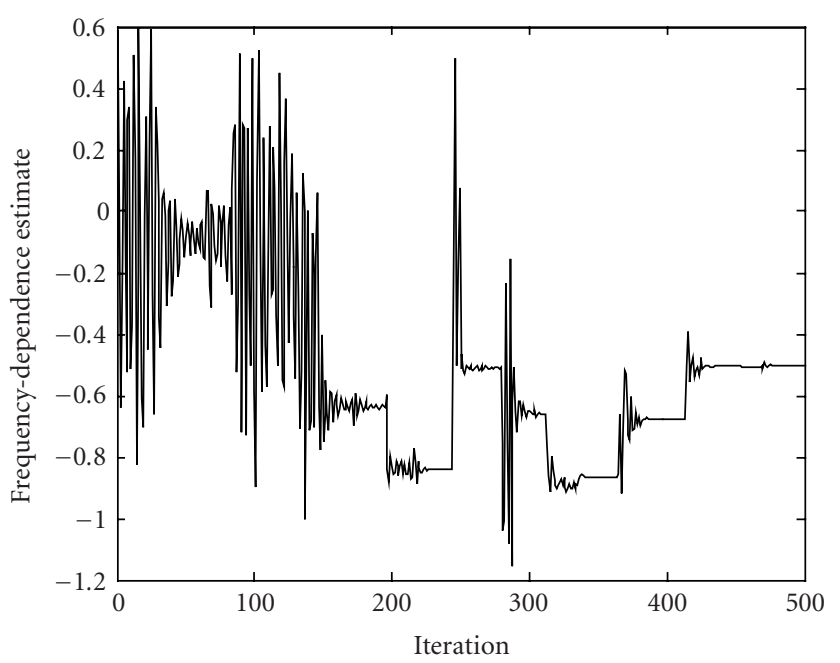

(c)

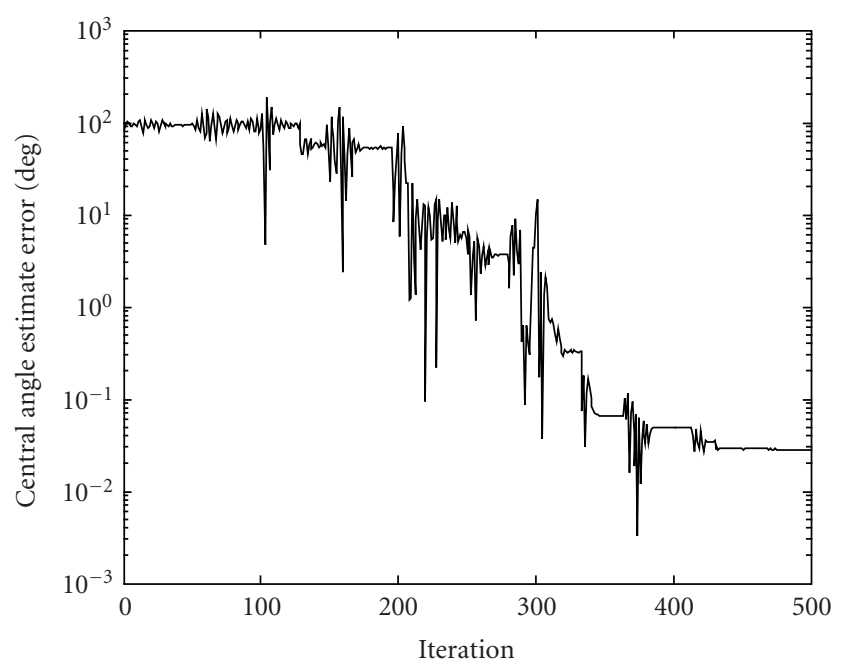

(b)

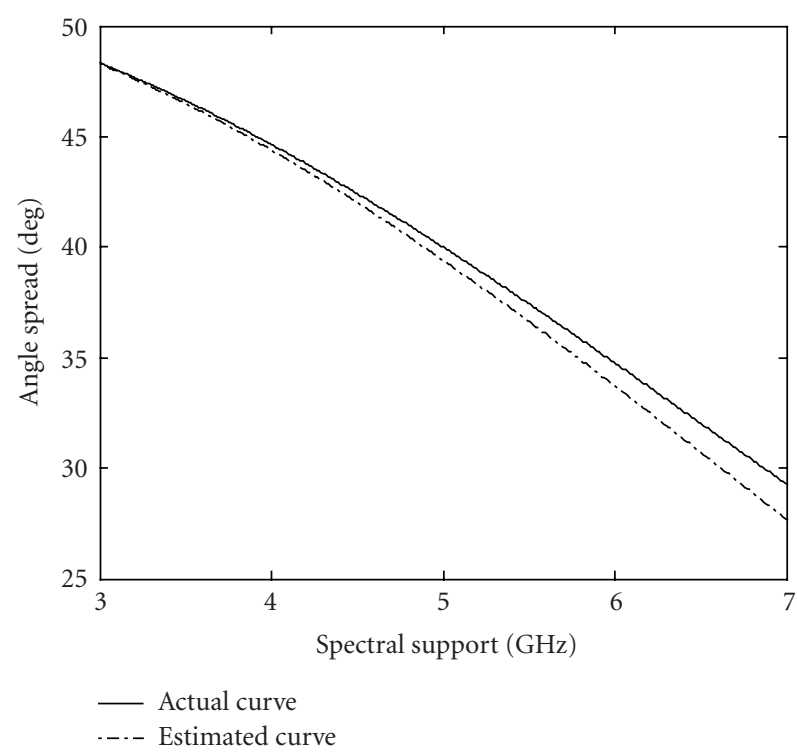

(d)

FIGURE 5: Convergence curves of (a) calculated fitness of the PSO algorithm, (b) central angle estimate error, (c) frequency-dependence estimate, and (d) the actual AS variation curve over the entire spectral support versus the estimated curve at the 500th iteration for Experiment 2 (Gaussian PAS).

normally distributed. From the plots of Figure 5, the proposed estimator yields a slightly deteriorated result in such a scenario in terms of the central angle estimate error and frequency-dependence estimate. Several other runs further testify the above observation. This is plausibly attributable to the fact that larger truncation errors are caused for Gaussian distributed signals when the fitness function (14) is evaluated by using numerical methods.

Experiment 3 (PAS shape mismatch). In this example, we investigate the effect of making a wrong assumption on the distribution of the spread signals. The scenario is identical to Experiment 2, in which a Gaussian distributed signal arrives at a mean DOA of $\underline{\theta}=\pi / 6$ to the normal of a uniform linear array. However, we assume that the signal is uniformly distributed and accordingly we apply (8) to (7) to calculate the signal covariance matrix and employ the particle-swarmoptimized Capon estimator to estimate the parameters.

Figure 6a depicts an irreducible fitness discrepancy because of the model mismatch. We can also observe from Figures $6 \mathrm{~b}$ and $6 \mathrm{c}$ that the performance of the algorithm has gone down significantly due to the wide signal spread. Another observation is that the PSO's convergence stalls at an earlier stage as compared to the previous two examples, 


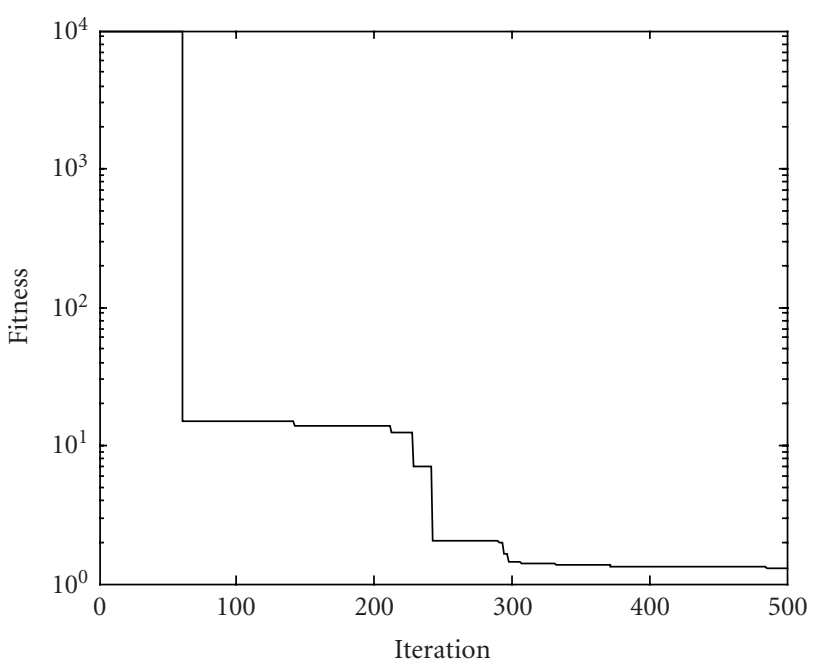

(a)

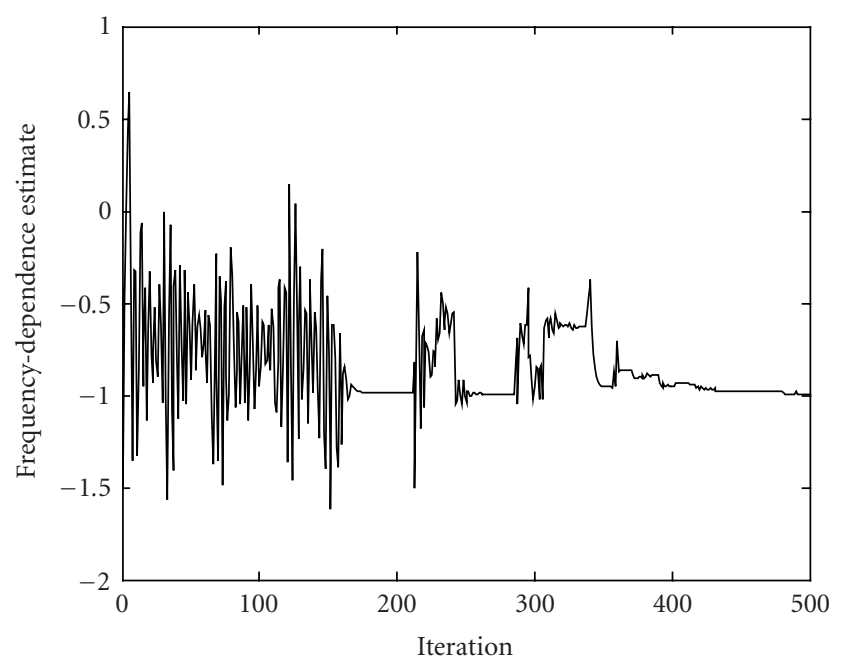

(c)

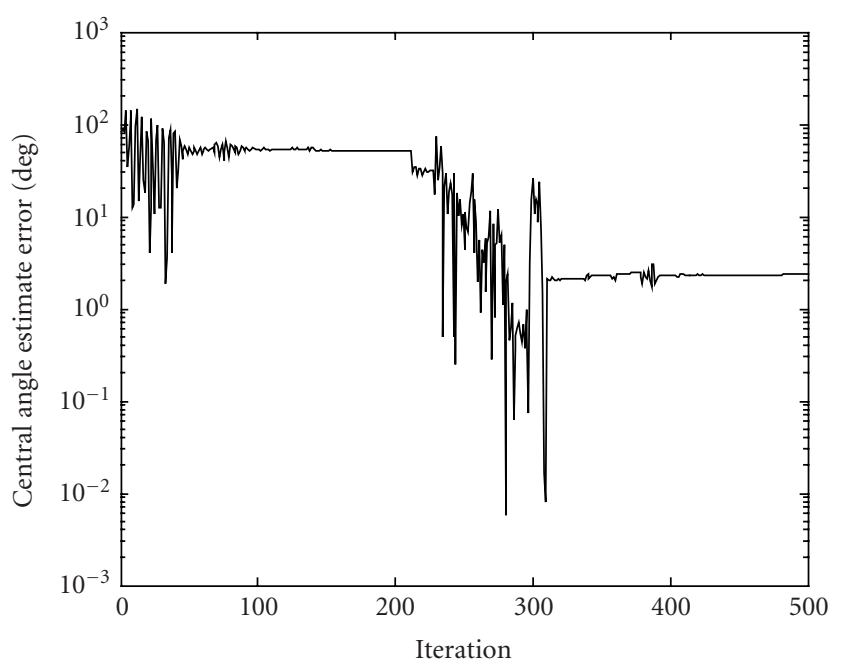

(b)

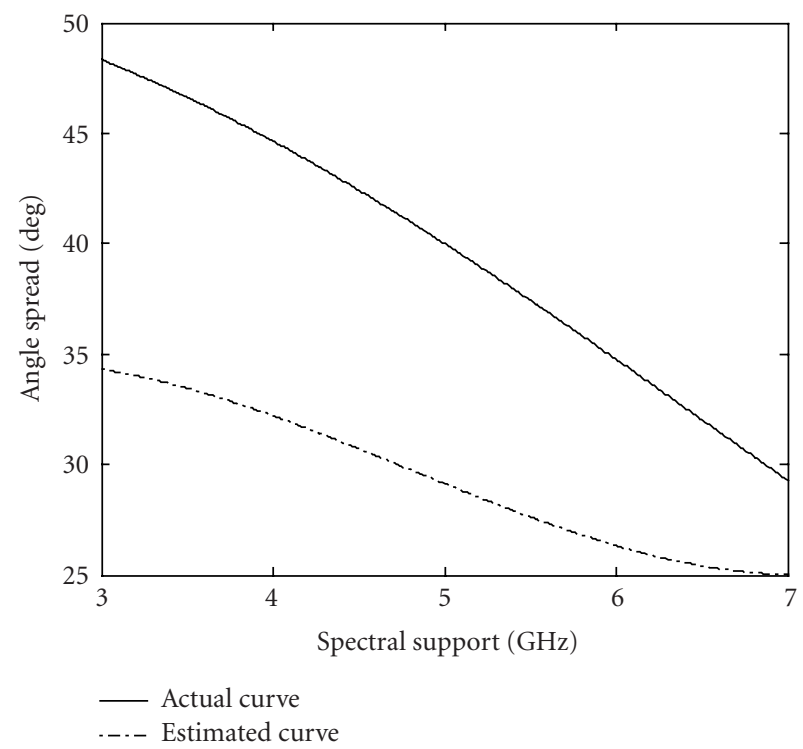

(d)

FIGURE 6: Convergence curves of (a) calculated fitness of the PSO algorithm, (b) central angle estimate error, (c) frequency-dependence estimate, and (d) the AS variation curve over the entire spectral support versus the estimated curve at the 500th iteration for Experiment 3 (PAS shape mismatch).

which shows that the original small optimal region (volume of the search space surrounding the global optimum) is replaced by a much larger pseudooptimal region surrounding many global optima leading to faulty results.

Experiment 4 (AS variation order mismatch). We continue to study the effect of making a wrong assumption on the order of the polynomial model for AS variation over the entire spectral support. This time the signal is uniformly distributed. The scenario is identical to that in Experiment 1, in which the actual AS variation curve has the form of a thirdorder polynomial. However, we assume that the AS variation can be best fit by polynomial regressions of orders $0,1,2$, and 3 in the PSO algorithms. As a result of increase of the dimensions, the population sizes are selected to be $20,50,80$, and 500 , respectively.

Figure $7 \mathrm{a}$ shows that the probability of generating a sample inside the feasible and optimal region decreases as the dimensionality of the search space increases. It is clear that it becomes much harder to find the global optimum of a highdimensional problem. In general, by assuming a third-order polynomial model, a large number of iterations are wasted to search for the small isolated feasible island, regardless of the relatively large population size used. Nevertheless, due to the 


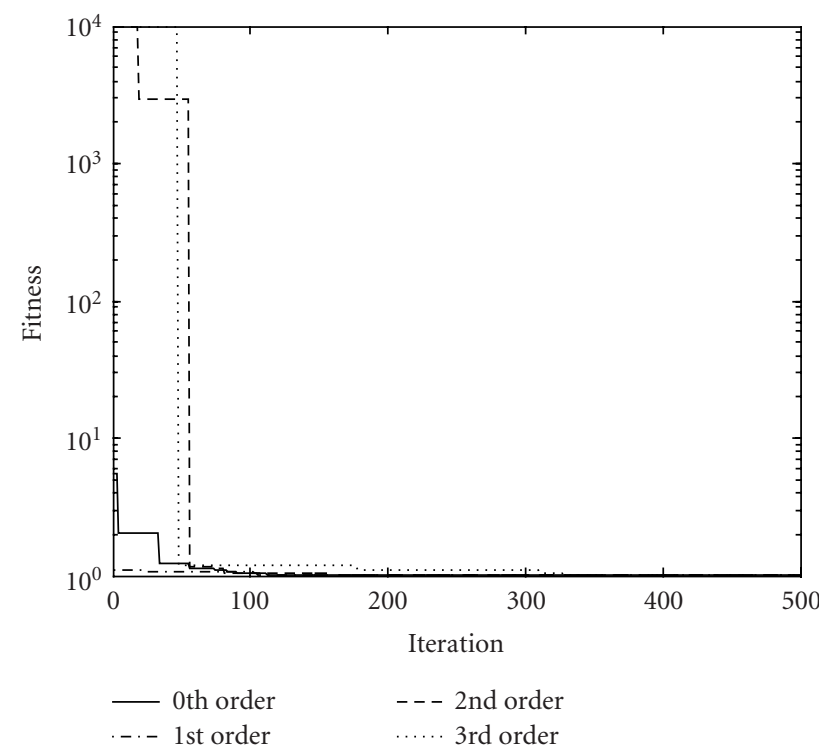

(a)

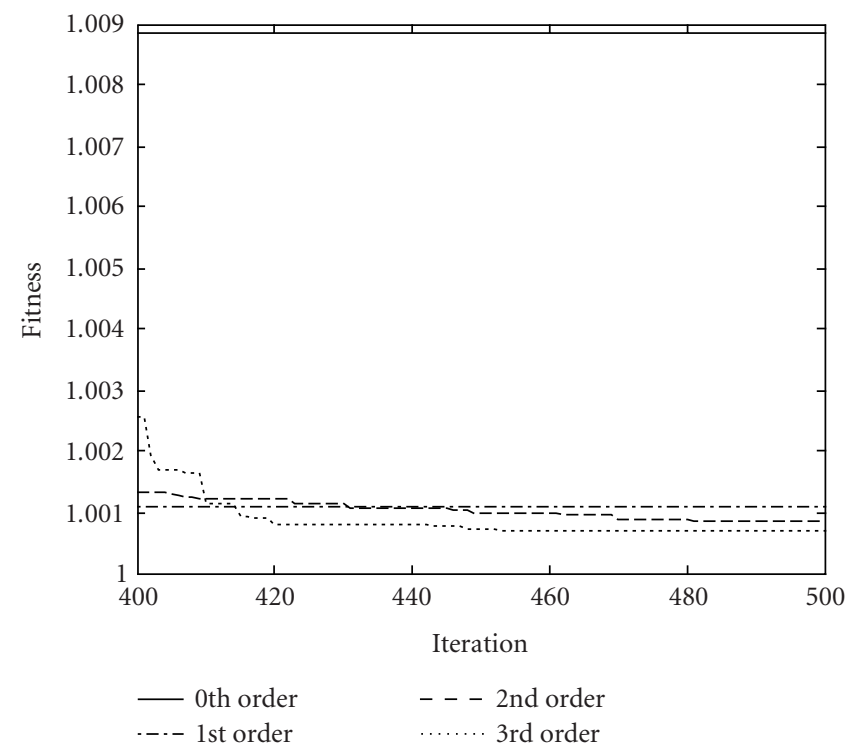

(b)

Figure 7: Convergence curves of calculated fitness of the PSO algorithm for Experiment 4 (AS variation order mismatch): (a) the original plot and (b) the zoomed-in plot.

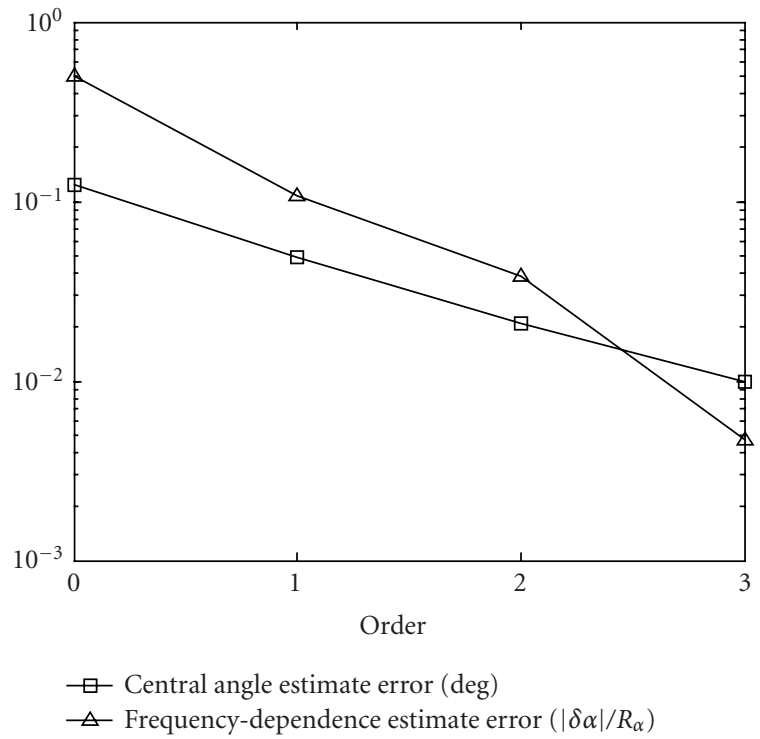

(a)

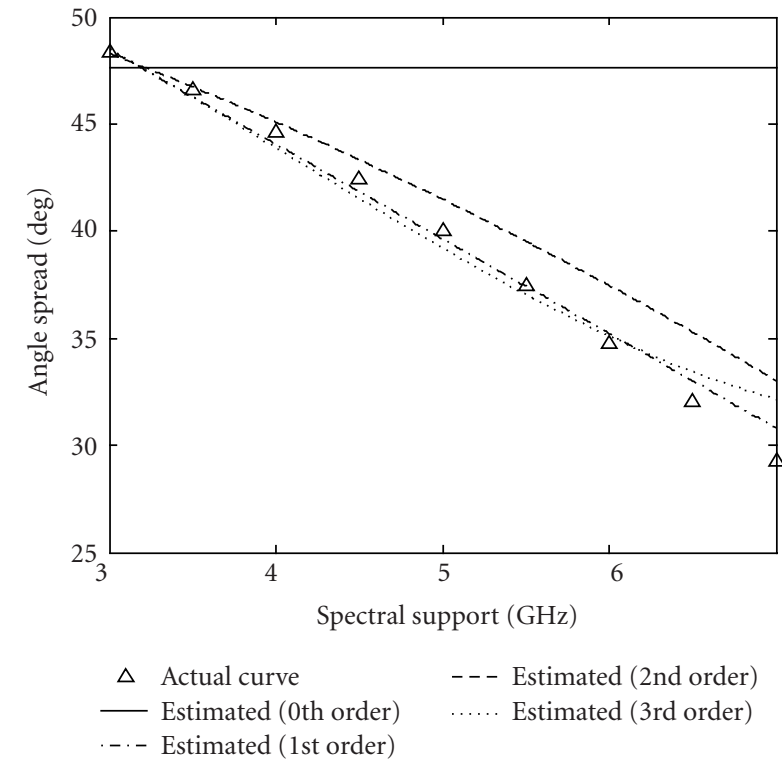

(b)

Figure 8: (a) Central angle and frequency-dependence estimate errors ( $\delta \alpha$ is the difference between the actual and estimated $\alpha$; $R_{\alpha}$ is the searching range) and (b) the actual AS variation curve over the entire spectral support versus the estimated curve at the 500th iteration for Experiment 4 (AS variation order mismatch).

model mismatch as we decrease the polynomial order, the attainable minimum fitness value increases, which is made apparent in Figure 7b. Therefore, it is important to choose an appropriate model dimension that maintains a good balance between the accuracy of the estimation results and the computational load. Figure 8 depicts that for the experiment presented here, the performance of the proposed estimator deteriorates as the model order decreases. In general, a large estimation error is resulted when the order is reduced to 0 , corresponding to the conventional narrowband wireless channel. Similar observations of significant deterioration in performance persist in several other runs. 


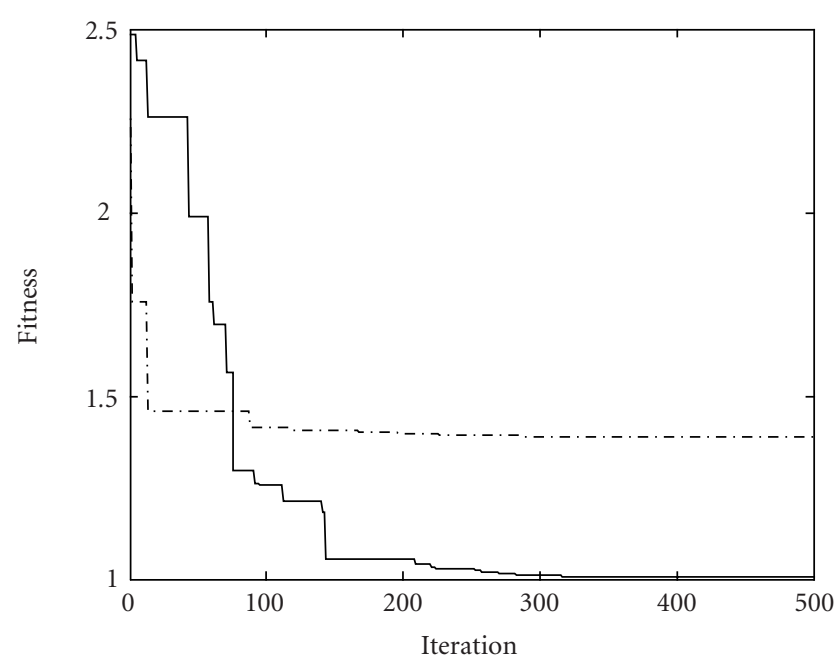

Without cluster number mismatch .... With cluster number mismatch

(a)

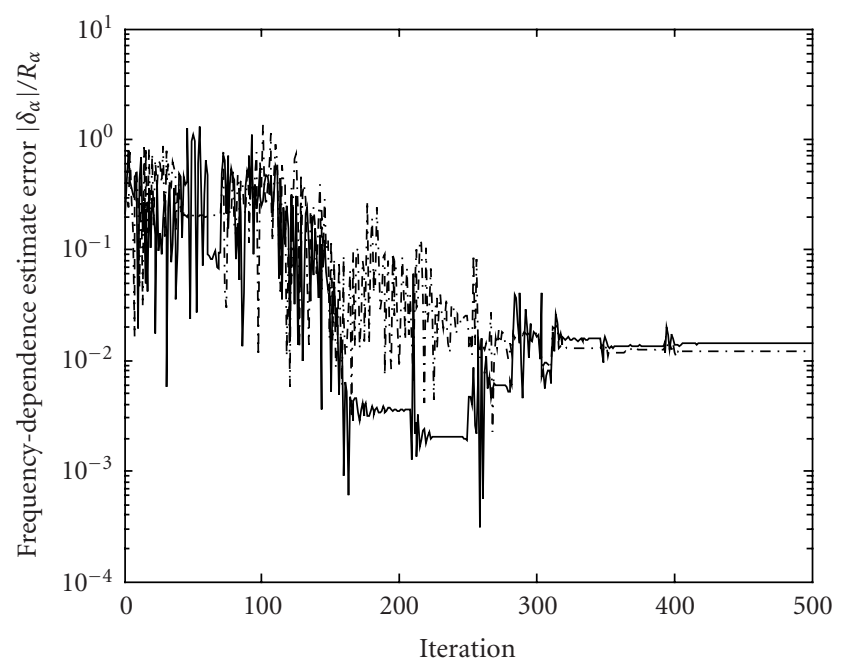

- Cluster 1

-..- Cluster 2

(c)

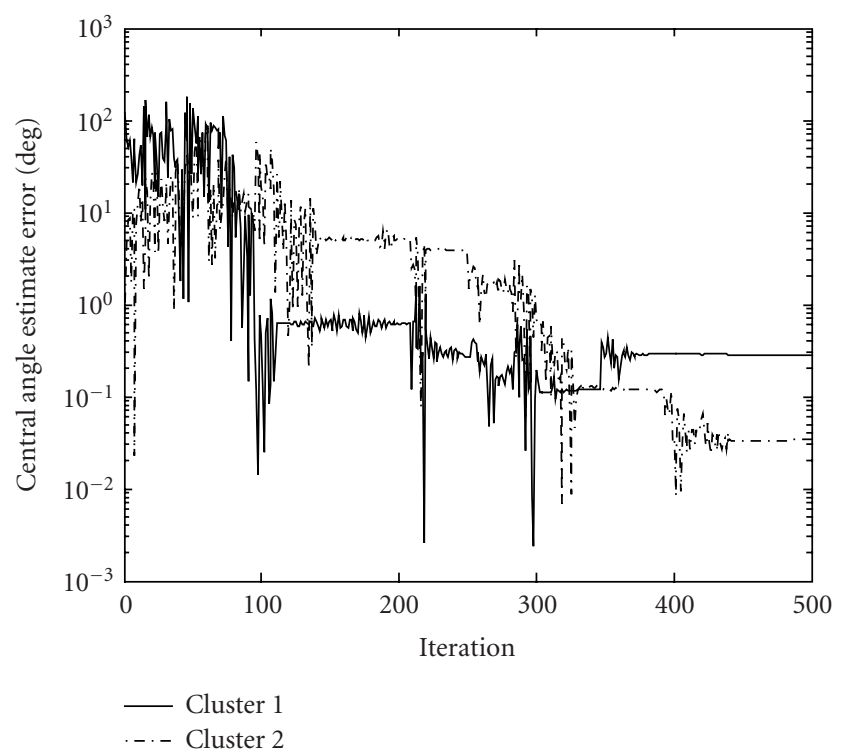

(b)

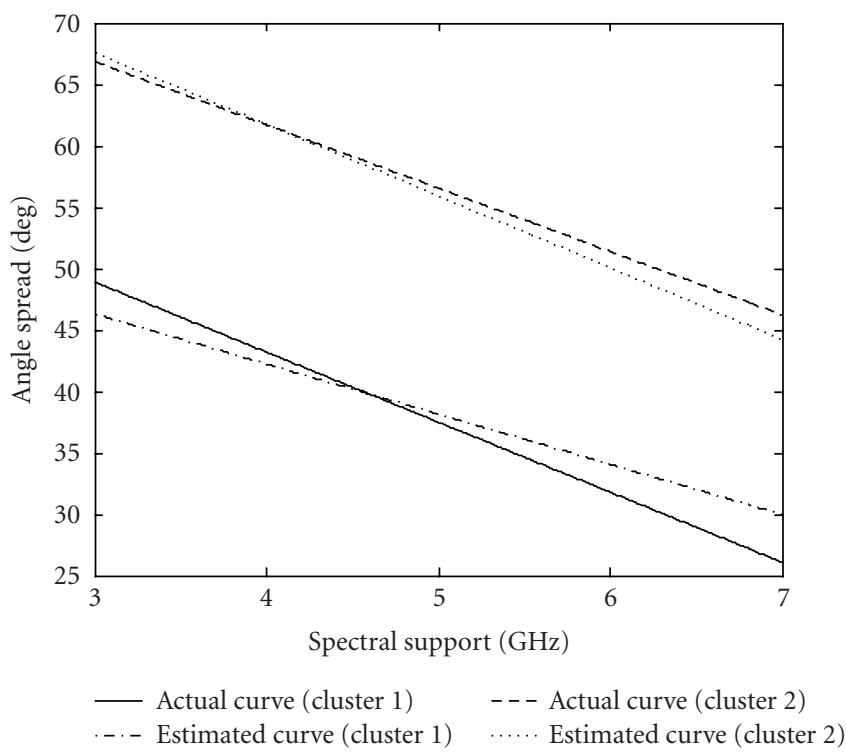

(d)

FIGURE 9: Convergence curves of (a) calculated fitness of the PSO algorithm, (b) central angle estimate error, (c) frequency-dependence estimate error, and (d) the actual AS variation curves over the entire spectral support versus the estimated curves at the 500th iteration for Experiment 5 (multicluster environments).

Experiment 5 (multicluster environments). In this experiment, we apply the similar procedure to estimate the angular information and frequency-dependence of two independent UWB sources, both uniformly distributed. It is assumed that the two scattering clusters are separated with $\underline{\theta}_{1}=-\pi / 6$ and $\underline{\theta}_{2}=\pi / 6$. Furthermore, the AS variation models take the form of the first-order polynomial. In addition, the frequency-dependence of the propagation modes can be described by $\alpha_{1}=-0.2$ and $\alpha_{2}=-0.5$. As such, it is assumed that the parameter vector to be estimated is $[-\pi / 6,-5.7,66,-0.2, \pi / 6,-5.15,82.3,-0.5]$ in an 8-dimensional hypercube. In the PSO algorithm, a population size of 80 is selected, $c_{1}$ and $c_{2}$ are both set to 2.0, and the inertial weight $w$ is varied linearly from 0.9 to 0.4 over 500 iterations.

From Figure 9a (solid line), it is clear that the particles swarm around the optimal region at around the 300th iteration. Figure $9 \mathrm{~b}$ shows the central angle estimate error versus the iteration number for the two clusters. We observe that the estimation accuracies are still acceptable when compared to 
the results in [17] for narrowband array signals with much narrower angle spread. Figure $9 \mathrm{c}$ shows that the frequencydependencies converge to the actual values at around the 400th iteration. Next, the estimated AS variation curves and the actual ones over the entire band are shown in Figure 9d where the estimated curves are obtained at the 500th iteration. Note that for this example the estimation is more accurate for cluster 2 when compared to the corresponding results for cluster 1. It is worth mentioning here that even when the actual AS variation curve can be better fit by higher-order polynomial equations, there would be moderate deterioration in the accuracy by assuming a 1st-order model.

Finally, we probe into the effect of making a wrong assumption on the number of scattering clusters. Figure 9a (dash-dotted line) illustrates that if the cluster number is mistakenly set to 1 , there will be an irreducible fitness floor, which is much larger than the expected minimum fitness value. This information is very useful when the number of clusters is unknown or inaccurate. By observing the minimum attainable fitness, it is possible to identify the exact number of clusters.

\section{CONCLUSIONS}

This paper introduced a new method for estimating the angular information of UWB sources and frequencydependence of the underlying propagation modes. The proposed method is based on a realistic description of UWB channel models: presence of local scattering, wideband array signals, and frequency-dependent dispersive effects. All of these facts have greatly complicated the estimation process and have not been jointly considered in the existing literature to the best of the authors' knowledge. Armed with the powerful and easy-to-implement PSO algorithms, the generalized Capon estimator has been extended to the UWB scenarios and applied for both the source localization and the structure frequency-dependence identification. Simulation results showed a satisfactory performance within a reasonable computation time for different environment types. The future work may include a more comprehensive investigation on the applicability of the modified PSO (e.g., objective-functiontransformed PSO and CPSO) on NP-hard UWB source localization problems (e.g., curse of dimensionality occurring in more complicated multicluster environments).

\section{REFERENCES}

[1] K. Siwiak and D. McKeown, Ultra-Wideband Radio Technology, John Wiley \& Sons, New York, NY, USA, 2004.

[2] M. G. M. Hussain, "Principles of space-time array processing for ultrawide-band impulse radar and radio communications," IEEE Trans. Veh. Technol., vol. 51, no. 3, pp. 393-403, 2002.

[3] H. D. Griffiths and A. L. Cullen, "Sidelobe response of antennas to short pulses - Part 1: Theory," IEE Proceedings - Microwaves, Antennas and Propagation, vol. 149, no. 4, pp. 189 193, 2002.

[4] R. Benjamin, "Sidelobe response of antennas to short pulses Part 2 Manifestations and applications," IEE Proceedings - Microwaves, Antennas and Propagation, vol. 149, no. 4, pp. 194199, 2002.
[5] R. J. Cramer, An evaluation of ultra-wideband propagation channels, Ph.D. dissertation, Electrical Engineering, University of Southern California, Los Angeles, Calif, USA, 2000.

[6] A. S. Y. Poon, Use of spatial dimension for spectrum sharing, $\mathrm{Ph} . \mathrm{D}$. dissertation, Electrical Engineering and Computer Sciences, University of California, Berkeley, Calif, USA, 2004.

[7] A. F. Molisch, J. R. Foerster, and M. Pendergrass, "Channel models for ultrawideband personal area networks," IEEE Wireless Communications, vol. 10, no. 6, pp. 14-21, 2003.

[8] "Spatio-temporal UWB propagation channel characterization," Communications Research Laboratory, Tokyo Institute of Technology and Tokyo Denki University, IEEE 802.15-040112-00-004a, March 2004.

[9] A. H. Muqaibel, Characterization of ultra wideband communication channels, Ph.D. dissertation, Electrical Engineering, Virginia Polytechnic Institute and State University, Blacksburg, Va, USA, 2003.

[10] R. C. Qiu, "A study of the ultra-wideband wireless propagation channel and optimum UWB receiver design," IEEE J. Select. Areas Commun., vol. 20, no. 9, pp. 1628-1637, 2002.

[11] R. C. Qiu and I. T. Lu, "Multipath resolving with frequency dependence for wide-band wireless channel modeling," IEEE Trans. Veh. Technol., vol. 48, no. 1, pp. 273-285, 1999.

[12] R. C. Qiu, Time/frequency dispersion of digital transmission media: wideband wireless channel model, chiral optical fiber, and superconducting MMIC, Ph.D. Dissertation, Polytechnic University, Brooklyn, NY, USA, 1996.

[13] R. C. Qiu, "A generalized time domain multipath channel and its application in ultra-wideband (UWB) wireless optimal receiver design: wave-based system analysis," to appear in IEEE Transactions on Wireless Communication, 2004.

[14] R. C. Qiu, "A generalized time domain multipath channel and its application in ultra-wide-band (UWB) wireless optimal receiver design: system performance analysis," in Proc. IEEE Wireless Communications and Networking Conference (WCNC '04), vol. 2, pp. 901-907, Atlanta, Ga, USA, March 2004.

[15] IEEE 802.15 WPAN Low Rate Alternative PHY Task Group 4a (TG4a) Channel Model Subcommittee Reports, Online http://www.ieee802.org/15/pub/TG4a.html.

[16] T. Svantesson, "Physical channel modeling of multi-element antenna systems," Tech. Rep. R002/2001, Department of Signals and Systems, Chalmers University of Technology, Göteborg, Sweden, 2001.

[17] A. Hassanien, S. Shahbazpanahi, and A. B. Gershman, "A generalized Capon estimator for localization of multiple spread sources," IEEE Trans. Signal Processing, vol. 52, no. 1, pp. 280283,2004

[18] J. Kennedy and R. Eberhart, "Particle swarm optimization," in Proc. IEEE International Conference on Neural Networks, vol. 4, pp. 1942-1948, Perth, Australia, November-December 1995.

[19] J. Kennedy, R. Eberhart, and Y. Shi, Swarm Intelligence, Morgan Kaufmann Publishers, San Francisco, Calif, USA, 2001.

[20] Y. Chen and V. K. Dubey, "UWB source localization using a particle-swarm-optimized Capon estimator," accepted for presentation in IEEE ICC 2005, Korea, 2004.

[21] M. Agrawal and S. Prasad, "Broadband DOA estimation using 'spatial-only' modeling of array data," IEEE Trans. Signal Processing, vol. 48, no. 3, pp. 663-670, 2000.

[22] R. B. Ertel, P. Cardieri, K. W. Sowerby, T. S. Rappaport, and J. H. Reed, "Overview of spatial channel models for antenna array communication systems," IEEE Pers. Commun., vol. 5, no. 1, pp. 10-22, 1998.

[23] Y. Meng, P. Stoica, and K. M. Wong, "Estimation of the directions of arrival of spatially dispersed signals in array processing," IEE Proceedings - Radar, Sonar and Navigation, vol. 143, no. 1, pp. 1-9, 1996. 
[24] M. Bengtsson and B. Ottersten, "Low-complexity estimators for distributed sources," IEEE Trans. Signal Processing, vol. 48, no. 8, pp. 2185-2194, 2000.

[25] J. Robinson and Y. Rahmat-Samii, "Particle swarm optimization in electromagnetics," IEEE Trans. Antennas Propagat., vol. 52, no. 2, pp. 397-407, 2004.

[26] D. W. Boeringer and D. H. Werner, "Particle swarm optimization versus genetic algorithms for phased array synthesis," IEEE Trans. Antennas Propagat., vol. 52, no. 3, pp. 771-779, 2004.

[27] J. Kennedy and W. M. Spears, "Matching algorithms to problems: an experimental test of the particle swarm and some genetic algorithms on the multimodal problem generator," in Proc. IEEE International Conference on Evolutionary Computation, pp. 78-83, Anchorage, Alaska, USA, May 1998.

[28] C. A. Coello Coello, G. T. Pulido, and M. S. Lechuga, "Handling multiple objectives with particle swarm optimization," IEEE Trans. Evol. Comput., vol. 8, no. 3, pp. 256-279, 2004.

[29] K. E. Parsopoulos and M. N. Vrahatis, "Recent approaches to global optimization problems through particle swarm optimization," Natural Computing, vol. 1, no. 2-3, pp. 235-306, 2002.

[30] P. N. Suganthan, "Particle swarm optimiser with neighbourhood operator," in Proc. IEEE Congress on Evolutionary Computation (CEC '99), vol. 3, pp. 1958-1962, Washington, DC, USA, July 1999.

[31] K. E. Parsopoulos and M. N. Vrahatis, "On the computation of all global minimizers through particle swarm optimization," IEEE Trans. Evol. Comput., vol. 8, no. 3, pp. 211-224, 2004.

[32] F. van den Bergh and A. P. Engelbrecht, "A cooperative approach to particle swarm optimization," IEEE Trans. Evol. Comput., vol. 8, no. 3, pp. 225-239, 2004.

Yifan Chen received the B.Eng. degree in electrical and electronic engineering with first-class honors from Nanyang Technological University (NTU), Singapore, in 2002. $\mathrm{He}$ is currently working toward the Ph.D. degree in the School of Electrical and Electronic Engineering, NTU, Singapore. His research interests are in the general areas of wireless communications, with emphasis on mobile radio channel stochastic modeling, ultrawide bandwidth signal propagation aspects, and multipleinput multiple-output channel characterization.

Vimal K. Dubey received the B.S. degree (with honors) in mathematics from the University of Rajasthan, India, the B.E. and M.E. degrees in electrical communication engineering from the Indian Institute of Science, Bangalore, India, and the Ph.D. degree in electrical engineering from McMaster University, Hamilton, Ontario, Canada. He has worked in various research and development laboratories in India for more

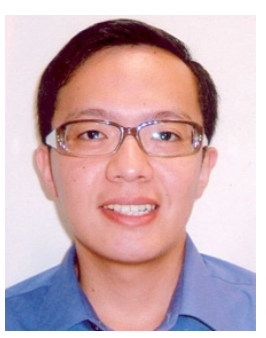
than ten years. From 1972 to 1976, he was a Research Scientist with DLRL, Hyderabad, India. From 1976 to 1982, he was with DEAL, Dehradun, India, where he conducted research on spread-spectrum systems for satellite communications and transportable troposcatter communication system. From 1982 to 1986, he was a Commonwealth Research Scholar at McMaster University.
He joined the School of Electrical and Electronic Engineering, Nanyang Technological University, Singapore, in 1988, where he is now an Associate Professor. His main research interests are in the areas of digital communications, specializing in coding, modulation, and spread-spectrum systems for satellite and wireless communications. 